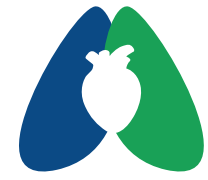

ASSOBRAFIR C I Ê N C I A

\section{Confiabilidade na identificação do limiar de anaerobiose ventilatória pelo método visual gráfico $^{\mathrm{a}}$}

\author{
Reliability analysis in the identification of the ventilatory \\ anaerobiosis threshold by the graphic visual method
}

Victor Regufe ${ }^{1,2}$ (D); Rafael Santiago Floriano 1,3 (D); Hugo Valverde Reis ${ }^{1,3}$ (D); Diogo Van Bavel ${ }^{1,2}$ (1); Michel Silva Reis ${ }^{1,2^{*}}$ (1)

\begin{abstract}
Resumo
Introdução: O Teste cardiopulmonar de exercício (TCPE) é o método padrão ouro na determinação de variáveis utilizadas na avaliação da capacidade funcional (CF). Um parâmetro bem consolidado é o limiar de anaerobiose ventilatório (LAV), que pode ser identificado por meio da análise visual das variáveis ventilatórias, sendo um método subjetivo. Objetivo: Analisar a confiabilidade na identificação do LAV pelo método visual gráfico por três observadores treinados e independentes diante de um banco de dados de TCPE. Métodos: Trata-se de um estudo, transversal e retrospectivo, realizado com 100 indivíduos de ambos os sexos (53 homens e 47 mulheres), com idade média de $49,77 \pm 16,55$ anos. Os valores de $\mathrm{VO}_{2}$ pico absoluto e relativo médios foram de 1,5 $\pm 0,8 \mathrm{~L} / \mathrm{min}$ e 19,0 $\pm 10,7 \mathrm{~mL} / \mathrm{Kg} / \mathrm{min}$, respectivamente. O TCPE foi realizado em cicloergômetro, com incremento de carga de 5 a $20 \mathrm{~W} / \mathrm{min}$, até a exaustão. Três avaliadores independentes realizaram a determinação do LAV, por meio da análise visual das variáveis ventilatórias. Resultados: Não houve diferença estatística entre as análises realizadas pelos avaliadores em nenhuma das variáveis. Foram encontradas correlações significativas fortes [tempo (s): $r=0,87 ; r=0,79 ; r=0,78$ ] e muito fortes $\left[\mathrm{VO}_{2}\right.$ relativo $(\mathrm{mL} / \mathrm{kg} / \mathrm{min})(\mathrm{r}=0,95 ; r=0,91 ; r=0,89)$ e $\mathrm{VO}_{2}$ absoluto $(\mathrm{L} / \mathrm{min})(r=0,94 ; r=0,90 ;$ $r=0,87)$ ] entre os avaliadores. Também foram encontradas concordâncias boas entre os avaliadores [tempo (s): 93\%; 94\%; 93\%; VO absoluto (L/min) (95\%; 89\%; 91\%; VO relativo (mL/kg/min): 92\%; 94\%; 94\%]. Conclusão: O método de análise visual por três observadores mostrou-se uma ferramenta confiável para identificar o LAV nos testes analisados.
\end{abstract}

Palavras-chave: Teste Cardiopulmonar de Exercício; Limiar Anaeróbio; Capacidade Funcional.

\begin{abstract}
Background: The cardiopulmonary test (CPT) is the gold standard method to determine several variables used in measurement functional capacity (FC). A well-established parameter is the ventilatory anaerobiosis threshold (VAT), which can be identified by visual analysis of ventilatory variables, which make this method subjective. Aim: To analyze the reliability in identifying the LAV by the visual graphic method by three trained and independent evaluators in front of a TCPE database. Methods: A cross-sectional and retrospective study was performed. The trial included 100 individuals of both genders (53 men and 47 women), mean age of $49.77 \pm 16.55$ years. The mean absolute and relative peak $\mathrm{VO}_{2}$ values were $1.5 \pm 0.8$ $\mathrm{L} / \mathrm{min}$ and $19.0 \pm 10.7 \mathrm{~mL} / \mathrm{Kg} / \mathrm{min}$, respectively. TCPE was performed on a cycle ergometer, load increase between 5 and $20 \mathrm{~W} / \mathrm{min}$, until exhaustion. Three independent evaluators determined the LAV, via visual analysis of the following ventilatory variables. Results: There was no statistical difference between the analysis performed by the evaluators in any variable. The correlations found among evaluators were significantly strong [time $(s)$ : $r=0.87 ; r=0.79$; $r=0.78$ ] and very strong [relative $\mathrm{VO}_{2}(\mathrm{~mL} / \mathrm{kg} / \mathrm{min}): \mathrm{r}=0.95 ; \mathrm{r}=0.91 ; \mathrm{r}=0.89$ and absolute $\mathrm{VO}_{2}$ (L/min): $r=0.94 ; r=0.90 ; r=0,87]$. There was also agreement between the evaluators [time (s): 93\%; 94\%; 93\%; absolute $\mathrm{VO}_{2}(\mathrm{~L} / \mathrm{min})$ (95\%; 89\%; 91\%; relative $\mathrm{VO}_{2}(\mathrm{~mL} / \mathrm{kg} / \mathrm{min}): 92 \%$; 94\%; 94\%]. Conclusion: This study showed that visual analysis method as a reliable tool to identify the VAT in the analyzed tests.
\end{abstract}

Keywords: Exercise Test; Anaerobic Threshold; Functional Capacity.
Estudo realizado em: Universidade Federa do Rio de Janeiro, Rio de Janeiro, RJ, Brasil. Aprovação ética: CAAE

47813415.8.0000.5257 do Hospital Universitário Clementino Fraga Filho da Universidade Federal do Rio de Janeiro, $\mathrm{n}^{\circ}$ 3.815 .491

*Autor correspondente: Michel Silva Reis E-mail: msreis@hucff.ufr..br
aApresentação dos dados em evento: Paulo em julho de 2020 e Congresso de HV, Bavel DV, Reis MS. Confiabilidade na ventilatóriça do limiar de anaerobiose ASSOBRAFIR Ciênc. 2021:12:e42366. http://

Submissão em: Dezembro 22, 2020

Aceito em: Outubro 18, 2021

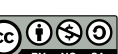




\section{INTRODUÇÃO}

O teste cardiopulmonar de exercício (TCPE) associado a um sistema de ergoespirometria é o padrão-ouro para determinar a capacidade funcional (CF) dos indivíduos, uma vez que nos permite acompanhar em tempo real as variáveis ventilatórias correspondentes aos ajustes metabólicos decorrentes do exercício ${ }^{1,2}$. Dentre essas variáveis, destacam-se o consumo de oxigênio $\left(\mathrm{VO}_{2}\right)$ e os limiares ventilatórios ${ }^{3-5}$.

O primeiro limiar ventilatório ou limiar de anaerobiose ventilatória (LAV) representa o momento de inversão do predomínio metabólico, durante um exercício incremental, coincidindo com o acúmulo sustentado de lactato muscular, induzindo um aumento da resposta ventilatória para manter o tamponamento isocápnico, com aumento da ventilação e metabolismo energético predominantemente anaeróbio ${ }^{2,6}$. Deste modo, o LAV pode ser considerado um bom indicador do condicionamento aeróbio.

A determinação do LAV pode ser realizada com base na interpretação do comportamento dos gases expirados. O equivalente ventilatório de oxigênio $\left(\mathrm{VE} / \mathrm{VO}_{2}\right)$ é a razão entre a taxa ventilatória instantânea e a taxa de captação de $\mathrm{O}_{2}$. Em um teste ou exercício incremental, essa variável tem sua curva inicialmente decrescente, em decorrência da baixa ventilação e do consumo crescente de $\mathrm{O}_{2^{\prime}}$ até atingir o menor valor, denominado nadir. Em seguida, com a elevação gradativa da intensidade, há uma transição do predomínio metabólico, ocorrendo um aumento da ventilação em detrimento do consumo de oxigênio $\left(\mathrm{VO}_{2}\right)$, sendo representado graficamente com elevação da curva VE/ $/ \mathrm{VO}_{2}{ }^{5,7}$.

Analisando-se os gases de forma isolada, observase que a fração expirada de oxigênio $\left(\mathrm{FEO}_{2}\right)$ tem o comportamento similar ao $\mathrm{VE} / \mathrm{VO}_{2}$, inicialmente decrescente, correspondendo ao aumento do $\mathrm{VO}_{2}$, o que leva a uma menor concentração do gás a nível da boca, até atingir o nadir e adotar um comportamento crescente, em decorrência da transição do predomínio metabólico. Portanto, pode-se concluir, que o nadir, em ambas as curvas, coincide com o LAV ${ }^{8,9}$.

Diante do exposto, a obtenção do LAV por meio da análise visual dos gráficos correspondentes às variáveis ventilatórias do TCPE, se mostra como um método não invasivo e prático, relacionado à tolerância ao exercício, pelo qual se pode estimar a CF, estratificar a aptidão física e a gravidade de doenças cardiorrespiratórias ${ }^{10-12}$ e para prescrever exercícios em um programa de reabilitação cardíaca ${ }^{7,8,13}$. No entanto, este método é subjetivo e avaliador dependente, fazendo-se necessária a avaliação de sua confiabilidade por diferentes observadores. Com isso, o objetivo do presente estudo foi analisar a confiabilidade na identificação do LAV pelo método visual por três avaliadores independentes. Desta forma hipotetizamos que a análise visual realizada por três avaliadores experientes no TCPE é confiável para a detecção e obtenção dos importantes marcadores ventilatórios e metabólicos são imprescindíveis para uma boa avaliação. Neste sentido fica claro que está avaliação assertiva irá gerar um diagnóstico diferencial e conseguente prescrição segura e individualizada de exercícios para as mais diversas finalidades que consiste este teste considerado o padrão ouro da avaliação da capacidade funcional.

\section{MÉTODOS}

\section{Desenho do estudo}

Trata-se de um estudo transversal e retrospectivo.

\section{Amostra}

A amostra consistiu em 162 TCPE, extraídos de um banco de dados de janeiro de 2017 a janeiro de 2019 do Grupo de Pesquisa em Avaliação e Reabilitação Cardiorrespiratória (GECARE/UFRJ). Os voluntários dos

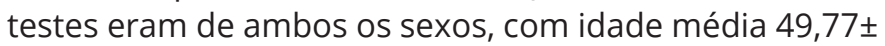
16,55 , incluindo indivíduos saudáveis, com fatores de risco para doença arterial coronariana (DAC), com Insuficiência Cardíaca e com Cirrose.

Foram incluídos no estudo, testes que apresentassem os seguintes critérios: executados em cicloergômetros verticais; potência incremental do tipo rampa; com duração entre 8 e 12 minutos; com taxa de amostragem de 10 segundos; captação com todos os parâmetros do TCPE presentes $\left(\mathrm{VO}_{2}, \mathrm{VCO}_{2}, \mathrm{VE}\right.$ e $\left.\mathrm{FEO}_{2}\right)$; do tipo máximo/sintomalimitado e com razão de troca gasosa (RER) maior que 1,0.

Foram excluídos os TCPE com dados incompletos, com ruídos frequentes que limitassem a análise e com respostas inadequadas (ausência do aumento fisiológico do $\mathrm{VO}_{2}$ com incremento da carga), resultando em 100 testes elegíveis para a análise.

Os dados analisados foram obtidos em um estudo prévios, e que foi aprovado pelo Comitê de Ética do Hospital Universitário Clementino Fraga Filho da UFRJ (CAAE 26421619.1.0000.5257). Todos os voluntários assinaram o Termo de Consentimento Livre e Esclarecido e a privacidade dos sujeitos da pesquisa e confidencialidade dos dados foram totalmente garantidas durante todas as etapas do estudo.

\section{Protocolo experimental}

Teste cardiopulmonar de exercício máximo ou sintoma limitado

O TCPE foi executado com protocolo do tipo rampa em cicloergômetro (BH- Ergocicle167, Belo Horizonte, MG, Brasil). O cálculo para o incremento de carga foi realizado de acordo com a CF relatada e a experiência do avaliador de modo que os testes durassem entre 8 e 12 minutos. 
Dessa maneira, a potência incremental foi definida entre 5-20 W/min. Os voluntários foram orientados a pedalar com uma cadência de 60-70 rpm.

Inicialmente, os voluntários permaneceram sentados no cicloergômetro em repouso durante um minuto. Na sequência, iniciaram o período de aquecimento, por três minutos, pedalando com carga de $15 \mathrm{~W}$. O período de exercício iniciou com o incremento de carga (5-20 W/min), até a exaustão física, sendo esta distribuição de carga controlada pelo avaliador junto ao sistema de ergometria. Por fim, o período de recuperação pós-teste, consistiu de três minutos na carga de 15 ou $25 \mathrm{~W}$ (de acordo com a carga máxima alcançada), seguidos de dois minutos de repouso, em que continuaram sentados no cicloergômetro.

A FC foi captada e registrada durante todo o período do teste. O eletrocardiograma ECG (Wincardio USB, Micromed, Brasília, Brasil.) - nas derivações MC5, DII, DIII, aVR, aVL e aVF modificadas e de V1 a V6 - foi monitorizado continuamente durante todo o procedimento experimental. A pressão arterial sistêmica (PA) foi verificada a cada três minutos, simultaneamente à percepção subjetiva de esforço central e muscular periférico de MMII por meio da Escala de Borg modificada (CR 0-10) ${ }^{14}$.

As variáveis ventilatórias e metabólicas foram obtidas por meio de um sistema computadorizado de análise ergoespirométrica $\left(\mathrm{VO}_{2000}\right.$ - Portable Medical Graphics Corporation ${ }^{\circledR}$ ). O volume expirado (VE) foi obtido por meio de um pneumotacômetro de Pitot de alto fluxo, conectado ao sistema $\mathrm{VO}_{2000}$ e acoplado a uma máscara facial, devidamente ajustada a fim de evitar que o ar escape. O voluntário foi instruído a não se comunicar de forma verbal, devendo apenas utilizar gestos manuais para informar o valor na escala de Borg, ou para sinalizar a interrupção do protocolo. O teste foi iniciado quando o RER se manteve próximo ao valor de 0,8 . $O$ equipamento fornece em tempo real os valores de $\mathrm{VO}_{2}, \mathrm{VCO}_{2}$ e $\mathrm{VE}$. Os valores de $\mathrm{VE} / \mathrm{VO}_{2}, \mathrm{VE} / \mathrm{VCO}_{2}$, as variáveis metabólicas, $\mathrm{RER}, \mathrm{FEO}_{2}, \mathrm{FECO}_{2}$ e a frequência respiratória (FR) também foram calculados e armazenados.

Os testes foram realizados em um ambiente com temperatura entre 22 e $24^{\circ} \mathrm{C}$ e com a umidade relativa do ar entre 50 e $70 \%$ e conduzidos por uma equipe de pesquisadores experientes, fisioterapeutas, médicos e profissionais de Educação Física, os quais estiveram atentos aos sinais e sintomas de resposta inadequada ao exercício. Foram utilizados os seguintes critérios de interrupção do teste: não manter a cadência de 6070 rotações por minuto; sudorese profusa, arritmias complexas (de alta frequência e multifocal), angina, palidez, FC máxima ${ }^{15}$, PA sistólica $>220 \mathrm{mmHg}$, PA diastólica > $110 \mathrm{mmHg}$, tontura, cianose e RER $\geq 1.10^{1}$.

\section{Método visual ventilatório}

As curvas da correlação $\mathrm{VO}_{2}$ e $\mathrm{VCO}$, relação $\mathrm{VE} / \mathrm{VO}_{2} \mathrm{e}$ $\mathrm{VE} / \mathrm{VCO}_{2}$, e das variáveis $\mathrm{FEO}_{2}$ e $\mathrm{FECO}$ foram plotadas em amostras de 10 segundos no software Excel. Na sequência, três observadores treinados - com experiência de no mínimo dois anos de participação na execução dos TCPE - e independentes avaliaram o comportamento das curvas e realizaram a determinação do LAV na ocorrência das seguintes situações: $\mathrm{VE} / \mathrm{VO}_{2}$ : ponto de mais baixo valor desta relação (nadir), certificando-se de que, a partir dele, ocorra aumento sistemático; $\mathrm{FEO}_{2}$ : ponto de mais baixo valor desta variável (nadir), a partir do qual, tem início uma elevação sistemática; valor de RER entre 0,9 e 1,0; perda de paralelismo entre $\mathrm{VO}_{2}$ e $\mathrm{VCO}_{2}$.

O trecho de análise selecionado foi com base nas respostas das variáveis cardiorrespiratórias, ou seja, do momento em que começam a responder ao incremento de potência até o momento de interrupção do exercício. A análise de cada observador foi realizada independentemente. A partir do valor médio do tempo obtido foram verificados os valores de potência, $\mathrm{VO}_{2}(\mathrm{~mL} /$ $\mathrm{min}), \mathrm{VO}_{2}(\mathrm{~mL} / \mathrm{kg} / \mathrm{min}$ ) e ventilação (VE) correspondentes na planilha das variáveis geradas pelo sistema de ergoespirometria, plotada a cada 10 segundos.

\section{Análise estatística}

Os dados foram analisados no programa estatístico Sigmaplot 11.0. Inicialmente, foram aplicados os testes de normalidade de Shapiro-Wilk e homogeneidade das variâncias de Levene para a determinação da distribuição da amostra. Em seguida, foi aplicado o Teste de Friedman com medidas repetidas e post hoc de Tukey para verificar a confiabilidade das medidas. Na sequência, foram realizadas Correlações de Pearson para avaliar a associação temporal e os valores de $\mathrm{VO}_{2}$ relativo e absoluto da determinação do LAV entre os três observadores (0-0,19correlação bem fraca; 0,20-0,39 - correlação fraca; 0,400,69 - correlação moderada; 0,70-0,89 - correlação forte; 0,90-1 correlação muito forte $)^{16}$. Por fim, foi realizado o teste de Bland-Altman, para verificar a concordância das medidas. As variáveis paramétricas foram apresentadas em média e desvio padrão e as não paramétricas em mediana e intervalos interquartis. O nível de significância estabelecido foi $p<0,05$.

\section{RESULTADOS}

Foram extraídos do banco de dados 162 TCPE. Destes, foram excluídos: 10 por terem sido executados em esteira rolante; 39 por serem testes de carga constante; 6 por estarem com ruídos frequentes ou incompletos; e 7 por não apresentarem o comportamento esperado das curvas, totalizando 100 dados elegíveis para análise ( $n=$ 100) (Figura 1).

A amostra foi composta por 53 homens e 47 mulheres; sendo 21 indivíduos saudáveis; 38 indivíduos com fatores de risco para Doença Arterial Coronariana; 31 indivíduos com Cirrose Hepática; e 10 indivíduos com Insuficiência 
Cardíaca. As características demográficas, antropométricas e clínicas podem ser vistas na Tabela 1.

A Figura 2 revela que não houve diferença significativa interavaliadores na determinação do LAV, analisando as variáveis tempo (s); $\mathrm{VO}_{2}$ absoluto ( $\left.\mathrm{L} / \mathrm{min}\right) ;$ e $\mathrm{VO}_{2}$ relativo ( $\mathrm{mL} / \mathrm{Kg} / \mathrm{min})$, confirmando a confiabilidade das medidas.

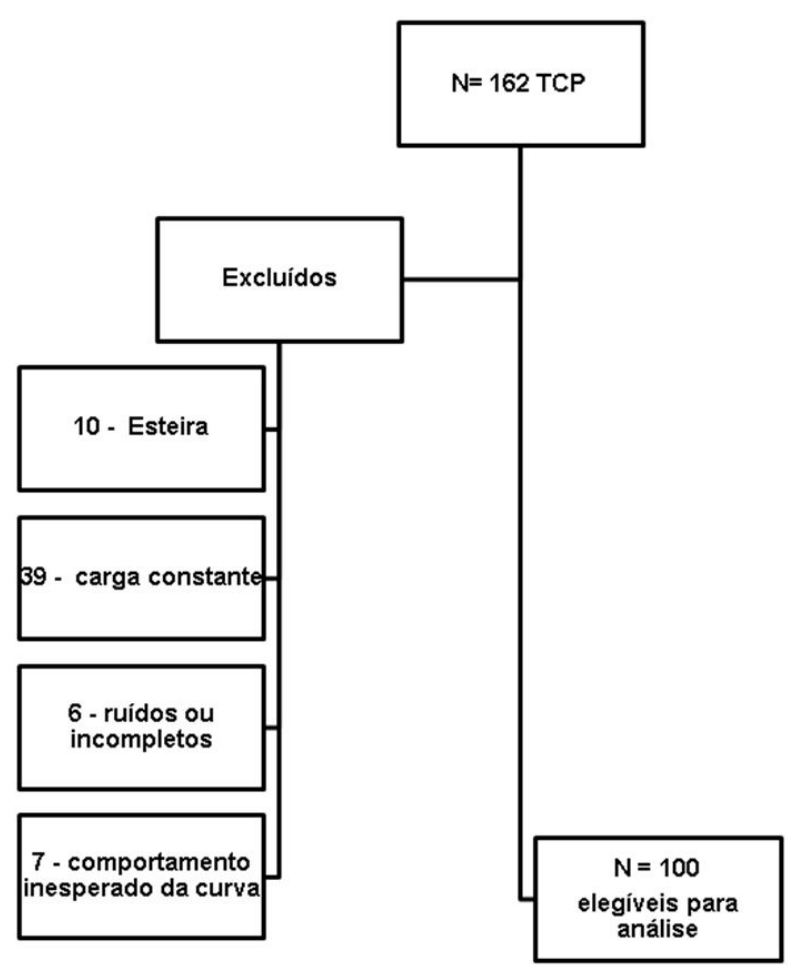

Figura 1. Fluxograma de seleção amostral. TCP: Teste Cardiopulmonar.
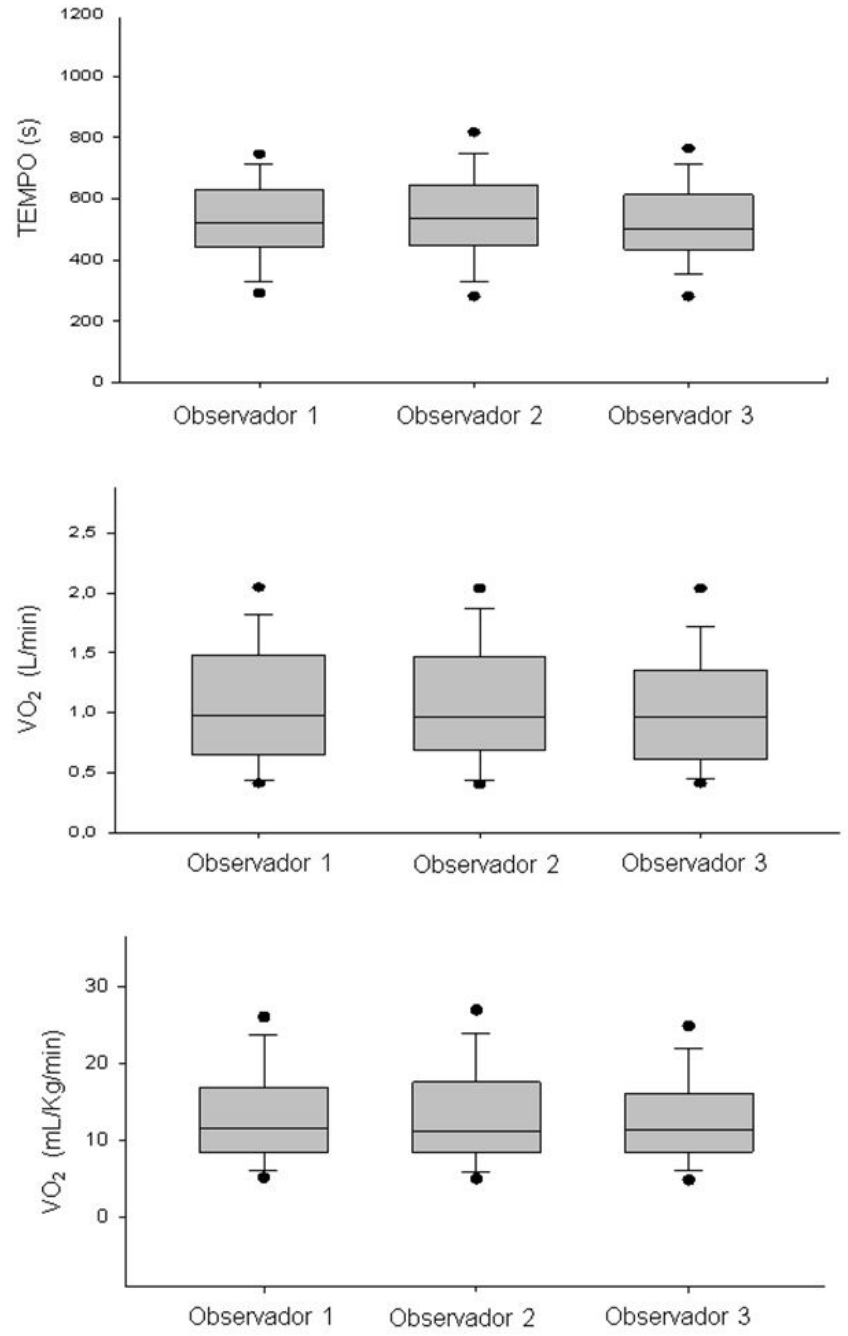

Figura 2. Teste de confiabilidade das medidas para os três avaliadores independentes (Teste de Friedman).

Tabela 1. Características gerais da amostra.

\begin{tabular}{|c|c|}
\hline Características gerais da amostra & $n=100$ \\
\hline \multicolumn{2}{|l|}{ Características demográficas } \\
\hline Idade (anos) & $49,77 \pm 16,55$ \\
\hline Sexo (masculino / \%) & $53 / 53$ \\
\hline \multicolumn{2}{|l|}{ Características antropométricas } \\
\hline Estatura (m) & $1,65 \pm 0,11$ \\
\hline Massa Corporal (kg) & $80,70 \mathrm{~kg} \pm 18,32$ \\
\hline IMC $\left(\mathrm{kg} / \mathrm{m}^{2}\right)$ & $29,37 \pm 5,39$ \\
\hline \multicolumn{2}{|l|}{ Características Clínicas } \\
\hline Saudáveis (n / \%) & $21 / 21$ \\
\hline Pacientes com fatores de risco para DAC (n / \%) & $38 / 38$ \\
\hline Pacientes com Insuficiência Cardíaca (n / \%) & $10 / 10$ \\
\hline Pacientes com Cirrose (n / \%) & $31 / 31$ \\
\hline \multicolumn{2}{|l|}{ Teste cardiopulmonar de exercício } \\
\hline $\mathrm{VO}_{2}$ pico (L/min) & $1,5 \pm 0,8$ \\
\hline $\mathrm{VO}_{2}$ pico $(\mathrm{mL} / \mathrm{kg} / \mathrm{min})$ & $19,0 \pm 10,7$ \\
\hline
\end{tabular}

Legenda: DAC: Doença Arterial Coronariana; IMC: Índice de massa corporal; $\mathrm{VO}_{2}$ : Consumo de Oxigênio. 
A Figura 3 mostra as correlações interavaliadores na determinação do LAV para a variável tempo, expressa em segundos (s). Foram obtidas correlações fortes $(r=0,87$ entre os observadores 1 e $2 ; r=0,79$ entre os observadores 1 e 3; e $r=0,78$ entre os observadores 2 e 3), todas significativas.

A Figura 4 mostra as correlações interavaliadores na determinação do LAV para o $\mathrm{VO}_{2}$ absoluto, expresso em litros por minuto (L/min). Foram obtidas correlações muito fortes ( $r=0,94$ entre os observadores 1 e 2; $r=0,90$ entre os observadores 1 e 3; e r=0,87 entre os observadores 2 e 3), todas significativas.
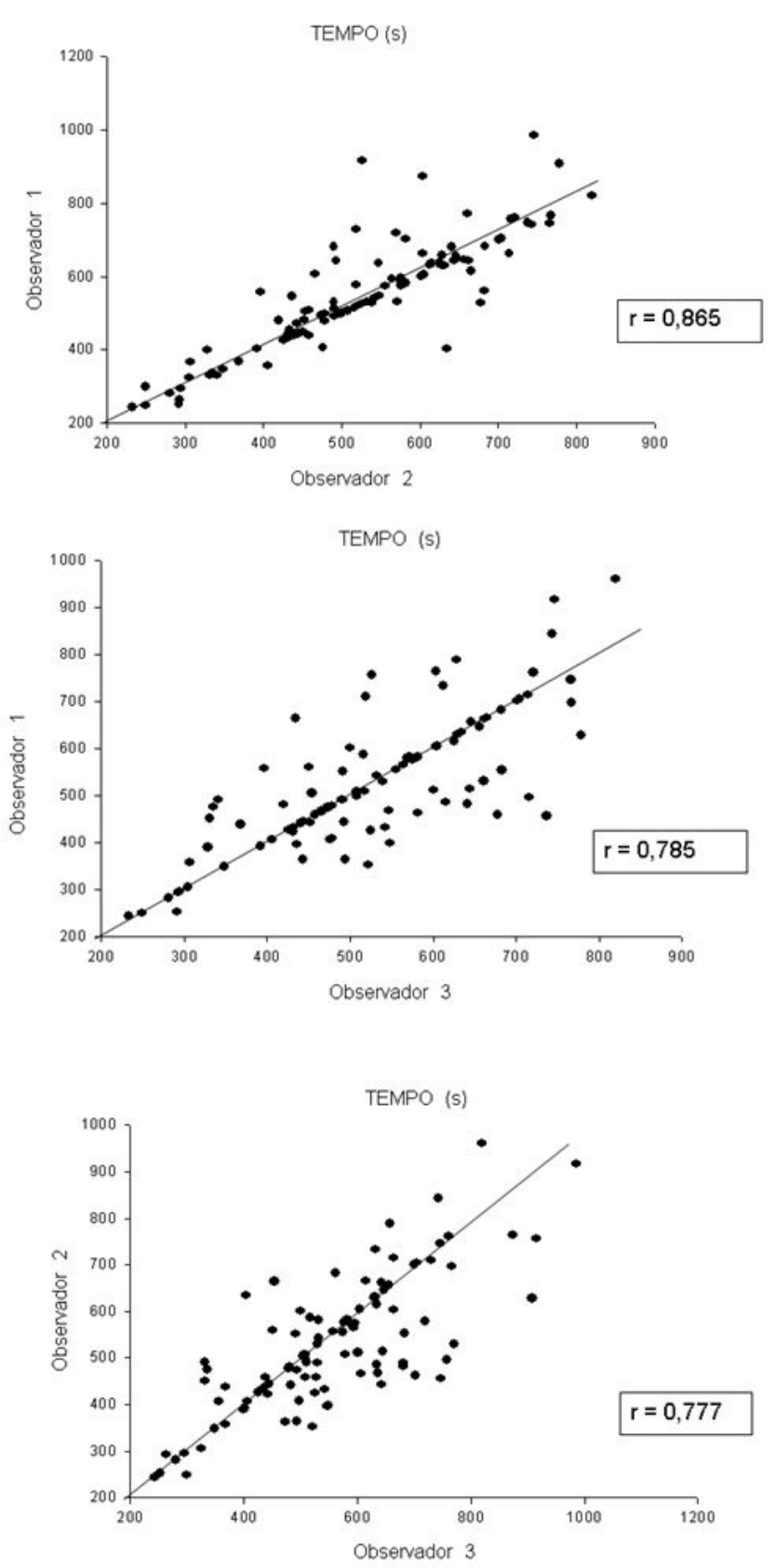

Figura 3. Correlações de Pearson entre as avaliações dos três observadores independentes para a variável tempo (s).
A Figura 5 mostra as correlações interavaliadores na determinação do LAV para a $\mathrm{VO}_{2}$ relativo, expresso em mililitros por quilogramas por minuto ( $\mathrm{mL} / \mathrm{Kg} / \mathrm{min})$. Foram obtidas correlações muito fortes ( $r=0,95$ entre os observadores 1 e 2; r=0,91 entre os observadores 1 e 3; e $r=0,89$ entre os observadores 2 e 3), todas significativas.

Por fim, foi realizada também a análise de BlandAltman interavaliadores na determinação do LAV. Foram observadas para a variável tempo, concordâncias de 93\%, 94\% e 93\% entre os avaliadores 1 e 2; 1 e 3; e 2 e 3 respectivamente (Figura 6). Já em relação ao $\mathrm{VO}_{2}$ absoluto, foram encontradas concordâncias de
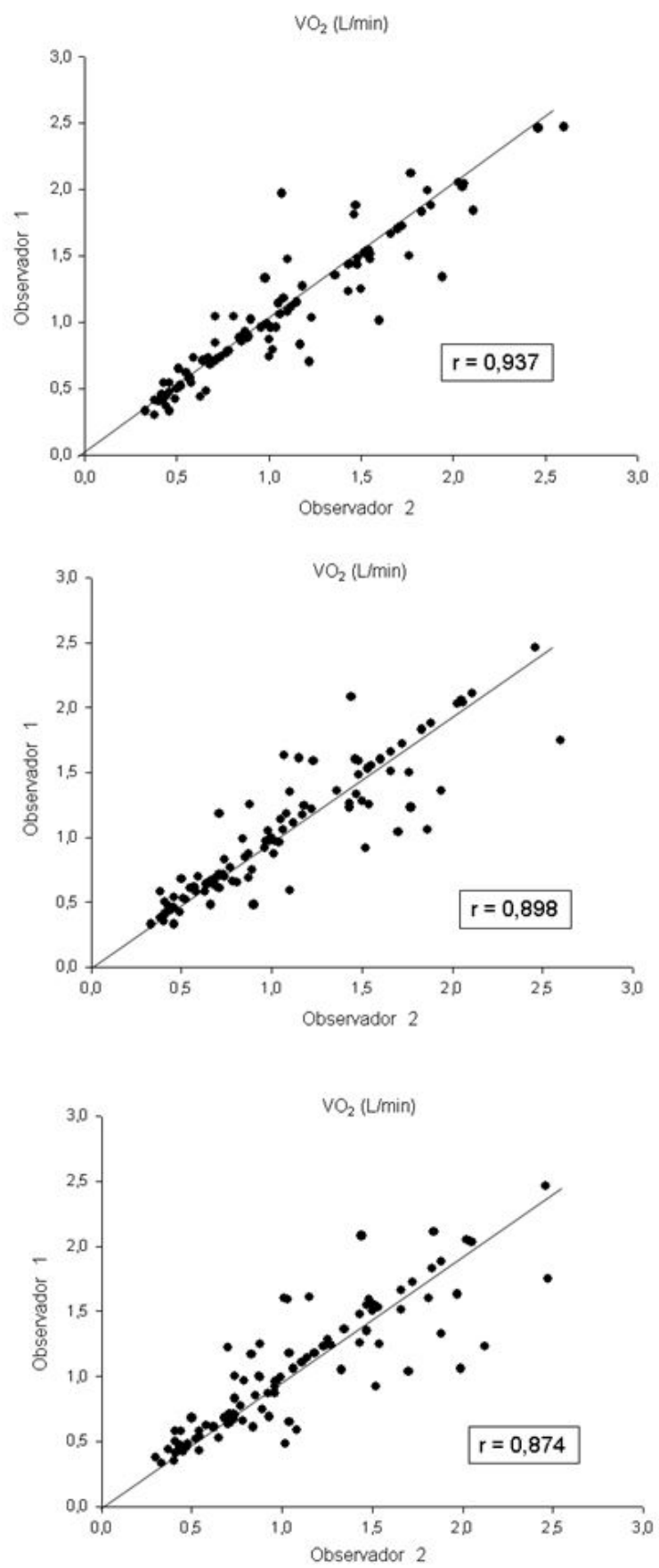

Figura 4. Correlações de Pearson entre as avaliações dos três observadores independentes para a variável $\mathrm{VO}_{2}$ absoluto (L/min). 
95\%, 89\% e 91\% entre os avaliadores 1 e 2; 1 e 3; e 2 e 3 respectivamente (Figura 7). Para o $\mathrm{VO}_{2}$ relativo, foram
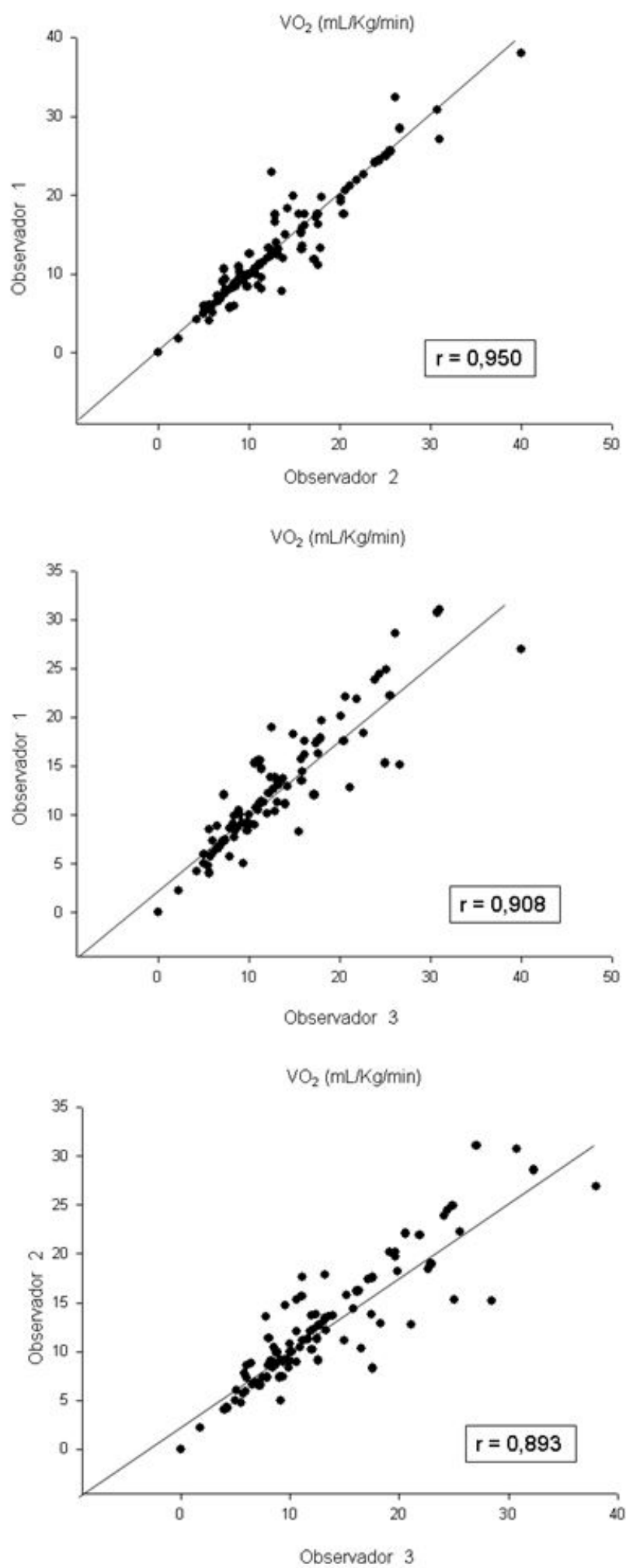

Figura 5. Correlações de Pearson entre as avaliações dos três observadores independentes para a variável $\mathrm{VO}_{2}$ relativo ( $\mathrm{mL} / \mathrm{Kg} / \mathrm{min})$. observadas concordâncias de 92\%, 94\% e 94\% entre os avaliadores 1 e 2; 1 e 3; e 2 e 3 respectivamente (Figura 8).
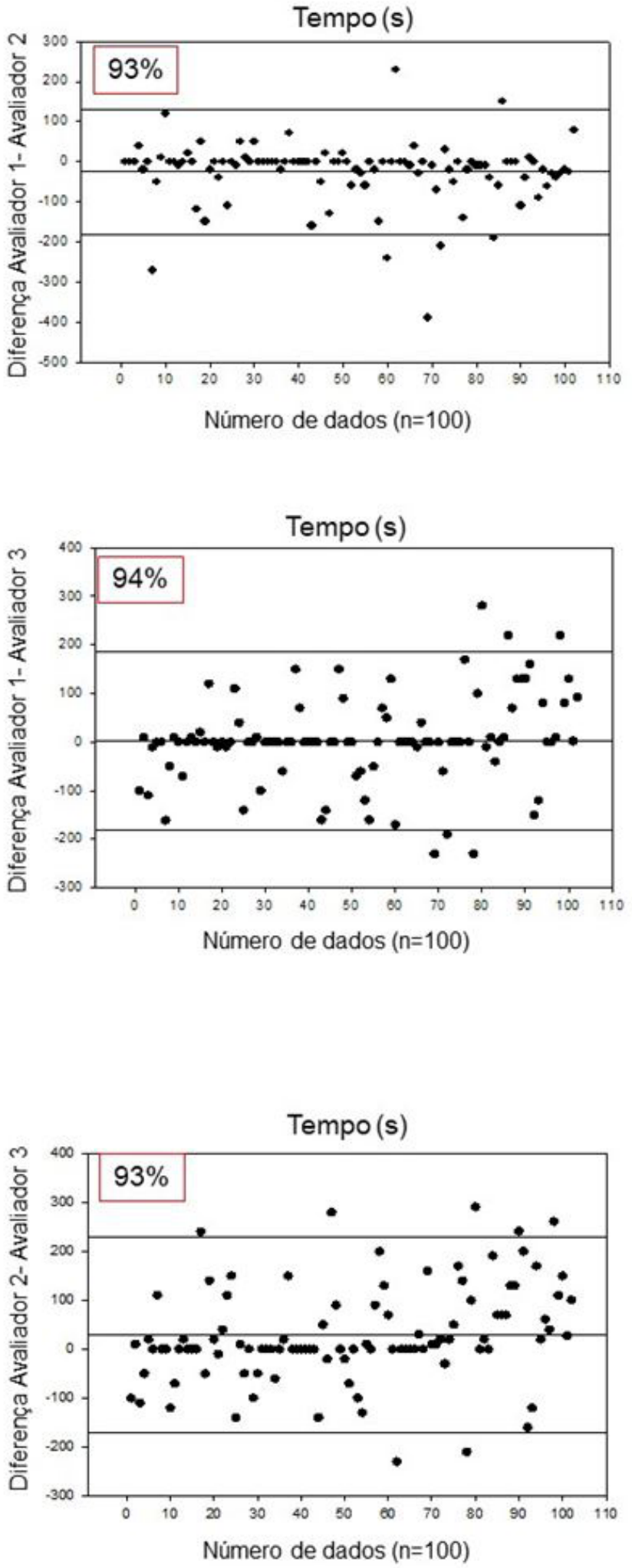

Figura 6. Teste de concordância de Bland-Altman para as avaliações dos três avaliadores independentes para a variável tempo. 

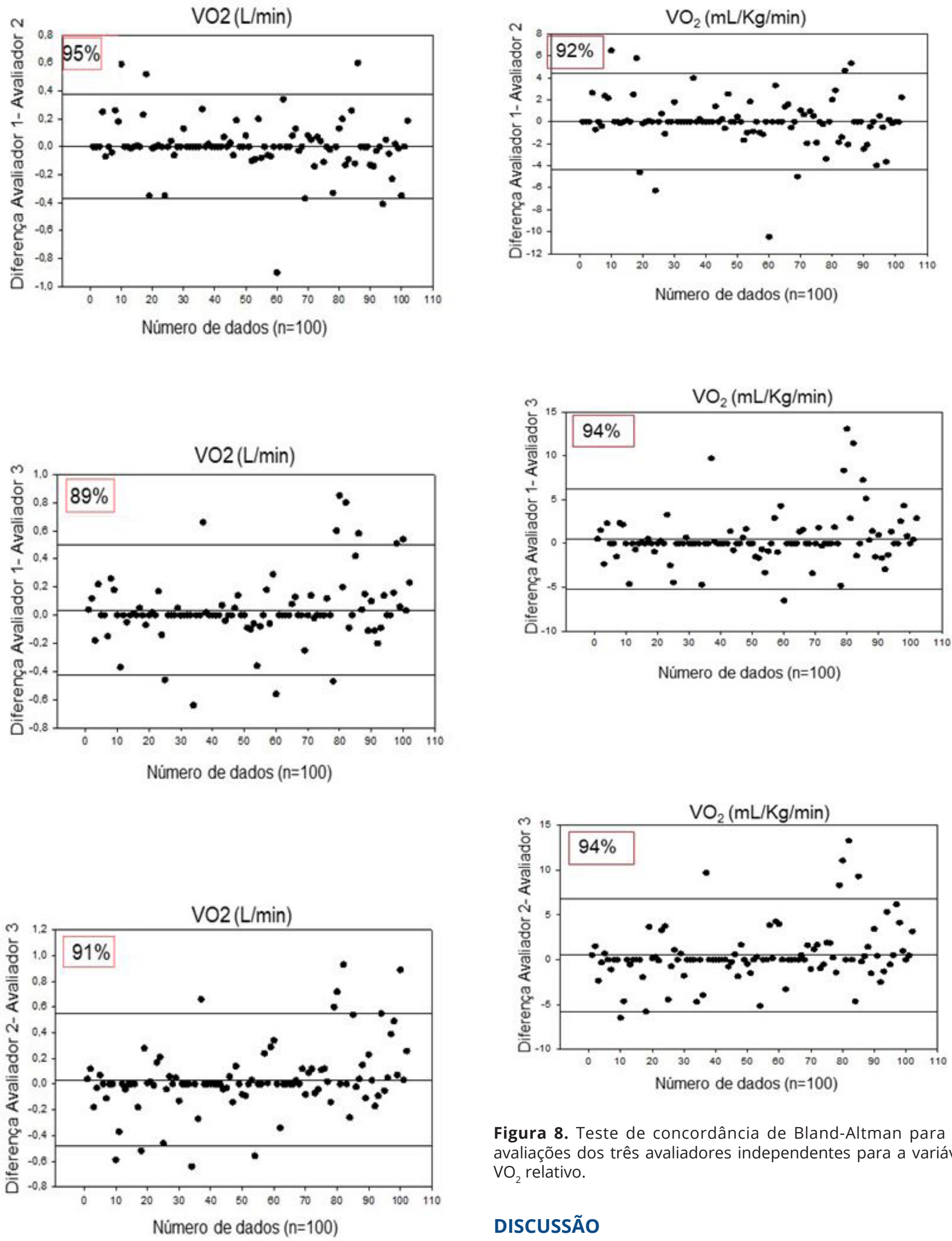

Figura 8. Teste de concordância de Bland-Altman para as avaliações dos três avaliadores independentes para a variável $\mathrm{VO}_{2}$ relativo.

\section{DISCUSSÃO}

O conceito de confiabilidade está relacionado à

Figura 7. Teste de concordância de Bland-Altman para as avaliações dos três avaliadores independentes para a variável $\mathrm{VO}_{2}$ absoluto. capacidade de reprodutibilidade de uma medida, variável ou dos resultados de uma determinada forma de avaliação ${ }^{16,17}$. O método de identificação do LAV de 
forma visual é subjetivo e avaliador-dependente, sendo assim suscetível a erros e/ou inconsistência no resultado. Por isso, fez-se necessário este estudo.

O limiar de anaerobiose é um fenômeno fisiológico que ocorre a nível muscular periférico e pode ser identificado diretamente por meio da lactacidemia em ratos $^{18,19}$ e em humanos ${ }^{20}$. Em contrapartida, a análise dos gases expirados durante o TCPE, como a realizada no presente estudo, é prática e não invasiva, apresentandose como uma alternativa segura para se determinar este fenômeno ${ }^{2,21}$. Entretanto, devemos considerar que há um atraso cinético entre a ocorrência do fenômeno a nível muscular e ventilatório ${ }^{22}$.

Diversas formas alternativas de determinação do LAV vêm sendo estudadas. Em idosos saudáveis, REIS et al. ${ }^{23}$ conseguiram identificar o LAV por meio da análise da saturação da hemoglobina diretamente na célula muscular, enquanto POZZI et al. ${ }^{24}$ elaboraram uma fórmula de predição, baseada na análise da frequência cardíaca (FC). LEPRÊTRE et al..$^{25}$ mostraram que a variabilidade da frequência cardíaca (VFC) pode ser um bom parâmetro para determinar o LAV em indivíduos com Insuficiência Cardíaca (IC), enquanto SIMÕES et al. ${ }^{26}$ mostraram que a VFC também tem boa correlação com a lactacidemia em indivíduos saudáveis.

Além disso, no presente estudo foi observado que a análise visual das variáveis ventilatórias se mostrou confiável e precisa, levando em consideração a heterogeneidade da amostra em questão. NISHIJIMA et al. ${ }^{27}$ realizaram um estudo similar, em que mostraram que o Vslope é confiável para identificar o LAV em populações heterogêneas. Contudo, para se representar graficamente o Vslope, é necessário realizar um cálculo de regressão. Além disso, há a hiperventilação decorrente da transição entre o aquecimento e o início de incremento de carga, que é considerado pelo software no momento do cálculo, podendo ser um fator confundidor. Já as curvas VE/VO $\mathrm{FEO}_{2}$ são representadas no próprio software durante o teste, podendo ser analisadas imediatamente após sua interrupção, não havendo necessidade de realizar novos cálculos.

\section{CONCLUSÃO}

Diante dos achados, pode-se concluir que a identificação do LAV pela análise visual gráfica das variáveis ventilatórias por três avaliadores independentes é um método confiável, prático e de fácil aplicação.

\section{FONTE DE FINANCIAMENTO}

Fundação Carlos Chagas de Apoio a Pesquisa do Estado do Rio de Janeiro e CNPq.

\section{CONFLITO DE INTERESSES}

Nada a declarar.

\section{REFERÊNCIAS}

1. Balady GJ, Arena R, Sietsema K, Myers J, Coke L, Fletcher GF, et al. Clinician's Guide to cardiopulmonary exercise testing in adults: a scientific statement from the American Heart Association. Circulation. 2010;122(2):191-225. http://dx.doi.org/10.1161/CIR.0b013e3181e52e69. PMid:20585013.

2. Floriano RS, Orsini M, Reis MS. Importância do teste cardiopulmonar para a fisioterapia cardiovascular. Fisioter Bras. 2019;20(4):578-91. http://dx.doi.org/10.33233/ fb.v20i4.2411.

3. Fenley A, Floriano RS, Chaves TO, Nasser I, Reis MS. Análise comparativa de fórmulas preditivas de avaliação da capacidade funcional com Teste cardiopulmonar de exercício de jogadoras de futebol profissional. Fisioter Pesqui. 2018;25(3):330-7. http://dx.doi.org/10.1590/18092950/17005725032018.

4. Thompson PD, Arena R, Riebe D, Pescatello LS. ACSM's new preparticipation health screening recommendations from ACSM's guidelines for exercise testing and prescription, ninth edition. Curr Sports Med Rep. 2013;12(4):215-7.

5. Neder JA, Nery LE. O teste de exercício cardiopulmonar. J Pneumol. 2002;28(3):166-206.

6. Neder JA, Nery LE. Fisiologia clínica do exercício: teoria e prática. São Paulo: Artmed 2003.

7. Herdy $A H$, Ritt LE, Stein R, Araújo CG, Milani $M$, Meneghelo RS, et al. Cardiopulmonary exercise test: background, applicability and interpretation. Arq Bras Cardiol. 2016;107(5):467-81. http://dx.doi.org/10.5935/ abc.20160171. PMid:27982272.

8. Mezzani A. Cardiopulmonary exercise testing: basics of methodology and measurements. Ann Am Thorac Soc. 2017;14(1):S3-S11.

9. Wasserman K, Hansen JE, Sue D, Whipp BJ, Casaburi R. Principles of exercise testing and interpretation. 3rd ed. Philadelphia: Lippincott Williams e Wilkins; 1999.

10. Carriere C, Corrà U, Piepoli M, Bonomi A, Merlo M, Barbieri $\mathrm{S}$, et al. Anaerobic threshold and respiratory compensation point identification during cardiopulmonary exercise tests in chronic heart failure. Chest. 2019;156(2):338-47. http:// dx.doi.org/10.1016/j.chest.2019.03.013. PMid:30926397.

11. Silva RRD, Reis MS, Pereira BB, Nascimento EMD, Pedrosa RC. Valor adicional do limiar anaeróbio em um modelo de predição de morte geral em uma coorte urbana de pacientes com cardiopatia chagásica. Rev Port Cardiol. 2017;36(12):927-34. http://dx.doi.org/10.1016/j. repc.2017.06.012. PMid:29223851.

12. Older $P$. Anaerobic threshold, is it a magic number to determine fitness for surgery? Perioper Med. 2013;2(1):2. http://dx.doi.org/10.1186/2047-0525-2-2. PMid:24472514.

13. Palermo $P$, Corrà U. Exercise Prescriptions for training and rehabilitation in patients with heart and lung disease. Ann Am Thorac Soc. 2017;14(1):S59-S66. http://dx.doi. org/10.1513/AnnalsATS.201702-160FR.

14. Borg G. Psychophysical scaling with applications in physical work and the perception of exertion. Scand J Work Environ Health. 1990;16(1):55-8. http://dx.doi.org/10.5271/ sjweh.1815. PMid:2345867. 
15. Karvonen MJ, Kentala E, Mustala O. The effects of training on heart rate; a longitudinal study. Ann Med Exp Biol Fenn. 1957;35(3):307-15. PMid:13470504.

16. Hopkins WG. Measures of reliability in sports medicine and science. Sports Med. 2000;30(1):1-15. http://dx.doi. org/10.2165/00007256-200030010-00001. PMid:10907753.

17. Toffoli SFL, Andrade DF, Bornia AC, Quevedo-Camargo G. Avaliação com itens abertos: validade, confiabilidade, comparabilidade e justiça. Educ Pesqui. 2016;42(2):343-58. http://dx.doi.org/10.1590/S1517-9702201606135887.

18. Abreu P, Mendes SVD, Leal-Cardoso JH, Ceccatto VM. Anaerobic threshold employed on exercise training prescription and performance assessment for laboratory rodents: a short review. Life Sci. 2016;151:1-6. http://dx.doi. org/10.1016/j.Ifs.2016.02.016. PMid:26860893.

19. Menezes HS, Coracini JCD, Kepler KC, Frantz E, Abegg MP, Correa CA, et al. Ácido láctico como indicativo de aptidão física em ratos. Rev Bras Med Esporte. 2010;3(16):210-4. http://dx.doi.org/10.1590/S1517-86922010000300011.

20. Wasserman K, Whipp BJ. Excercise physiology in health and disease. Am Rev Respir Dis. 1975;112(2):219-49. PMid:239617.

21. Kaczmarek S, Habedank D, Obst A, Dörr M, Völzke H, Gläser $S$, et al. Interobserver variability of ventilatory anaerobic threshold in asymptomatic volunteers. Multidiscip Respir Med. 2019;14(1):20. http://dx.doi.org/10.1186/s40248-0190183-6. PMid:31198557.

22. Sales MM, Sousa CV, da Silva Aguiar S, Knechtle B, Nikolaidis PT, Alves PM, et al. An integrative perspective of the anaerobic threshold. Physiol Behav. 2019;205:29-32. http:// dx.doi.org/10.1016/j.physbeh.2017.12.015. PMid:29248631.

23. Reis MS, Berton DC, Arena R, Catai AM, Neder JA, Borghi-Silva A. Determination of anaerobic threshold through heart rate and near infrared spectroscopy in elderly healthy men. Braz J Phys Ther. 2013;17(5):506-15. http://dx.doi.org/10.1590/ S1413-35552012005000115. PMid:24173351.

24. Pozzi LG, Melo RC, Quiterio RJ, Milan LA, Diniz CAR, Dias TCM, et al. Determinação do limiar de anaerobiose de idosos saudáveis: comparação entre diferentes métodos. Rev Bras Fisioter. 2006;10(3):333-8. http://dx.doi.org/10.1590/\$141335552006000300013.

25. Leprêtre PM, Ghannem M, Bulvestre $M$, Ahmaidi S, Delanaud S, Weissland T, et al. Exercise-based cardiac rehabilitation in coronary disease: training impulse or modalities? Int J Sports Med. 2016;37(14):1144-9. http:// dx.doi.org/10.1055/s-0042-112591. PMid:27832671.

26. Simões RP, Castello-Simões V, Mendes RG, Archiza B, Santos DA, Machado HG, et al. Lactate and heart rate variability threshold during resistance exercise in the young and elderly. Int J Sports Med. 2013;34(11):991-6. http://dx.doi. org/10.1055/s-0033-1337946. PMid:23606341.

27. Nishijima H, Kominami K, Kondo K, Akino M, Sakurai M. New method for the mathematical derivation of the ventilatory anaerobic threshold: a retrospective study. BMC Sports Sci Med Rehabil. 2019;11(1):10. http://dx.doi.org/10.1186/ s13102-019-0122-z. PMid:31285827. 